\title{
Providing Humanitarian Relief Support through Knowledge Graphs
}

\author{
Rui Zhu* \\ ruizhu@ucsb.edu \\ UC Santa Barbara, USA \\ Cogan Shimizu \\ coganmshimizu@ksu.edu \\ Kansas State University, USA \\ Anna Lopez-Carr \\ alopez-carr@directrelief.org \\ Direct Relief, USA \\ Yuanyuan Tian \\ ytian72@asu.edu \\ Arizona State University, USA
}

\author{
Ling Cai ${ }^{*}$ \\ lingcai@ucsb.edu \\ UC Santa Barbara, USA \\ Colby K. Fisher \\ cfish25@gmail.com \\ Hydronos Labs, USA \\ Andrew Schroeder \\ aschroeder@directrelief.org \\ Direct Relief, USA \\ Shirly Stephen \\ shirlystephen@ucsb.edu \\ UC Santa Barbara, USA
}

\author{
Gengchen Mai \\ maigch@cs.stanford.edu \\ Stanford University, USA \\ Krzysztof Janowicz \\ janowicz@ucsb.edu \\ UC Santa Barbara, USA \\ Mark Schildhauer \\ schild@nceas.ucsb.edu \\ UC Santa Barbara, USA \\ Zilong Liu
zilongliu@ucsb.edu \\ UC Santa Barbara, USA
}

\begin{abstract}
Disasters are often unpredictable and complex events, requiring humanitarian organizations to understand and respond to many different issues simultaneously and immediately. Often the biggest challenge to improving the effectiveness of the response is quickly finding the right expert, with the right expertise concerning a specific disaster type/disaster and geographic region. To assist in achieving such a goal, this paper demonstrates a knowledge graph-based search engine developed on top of an expert knowledge graph. It accommodates three modes of information retrieval, including a follow-your-nose search, an expert similarity search, and a SPARQL query interface. We will demonstrate utilizing the system to rapidly navigate from a hazard event to a specific expert who may be helpful, for example. More importantly, as the data is fully integrated including links between hazards and their abstract topics, we can find experts who have relevant expertise while navigating the graph.
\end{abstract}

\section{CCS CONCEPTS}

- Information systems $\rightarrow$ Search interfaces; Expert search; Ontologies; $\bullet$ Computing methodologies $\rightarrow$ Semantic networks.

\section{KEYWORDS}

Expert System, Expert Search, Knowledge Graph, Similarity, Disaster Response

\footnotetext{
*Both authors contributed equally to this research.

Permission to make digital or hard copies of all or part of this work for personal or classroom use is granted without fee provided that copies are not made or distributed for profit or commercial advantage and that copies bear this notice and the full citation on the first page. Copyrights for components of this work owned by others than ACM must be honored. Abstracting with credit is permitted. To copy otherwise, or republish, to post on servers or to redistribute to lists, requires prior specific permission and/or a fee. Request permissions from permissions@acm.org.

K-CAP '21, December 2-3, 2021, Virtual Event, USA.

(C) 2021 Association for Computing Machinery.

ACM ISBN 978-1-4503-8457-5/21/12 . \$ $\$ 15.00$

https://doi.org/10.1145/3460210.3493581
}

ACM Reference Format:

Rui Zhu, Ling Cai, Gengchen Mai, Cogan Shimizu, Colby K. Fisher, Krzysztof Janowicz, Anna Lopez-Carr, Andrew Schroeder, Mark Schildhauer, Yuanyuan Tian, Shirly Stephen, and Zilong Liu. 2021. Providing Humanitarian Relief Support through Knowledge Graphs. In Proceedings of the 11th Knowledge Capture Conference (K-CAP '21), December 2-3, 2021, Virtual Event, USA. ACM, New York, NY, USA, 4 pages. https://doi.org/10.1145/3460210.3493581

\section{INTRODUCTION}

Disasters are unpredictable, ever-changing, complex, and require quick responses to various, interdependent, simultaneous issues. For example, during the 2020 hurricane season, Hurricane Laura, a deadly and destructive Category 4 hurricane, hit the state of Louisiana. It caused a major power outage, water shortage, infrastructure failure, and extensive property damage in many areas. Evacuation and displacement strategies, which were historically effective, were less so due to the emergent COVID-19 pandemic, as people were less willing to relocate due to resurgent disease outbreaks. Even worse, six weeks later the Gulf Coast region was hit again by another storm, namely Hurricane Delta.

In order to react to such a dynamic and rapidly-evolving situation, relief organizations need to acquire and process information about the impacted regions, such as the damage to properties, recent (disaster) events, local population density, as well as similar historical disasters and their corresponding government responses (irrespective of historical co-location). Subsequently, relief specialists often connect to experts with particular knowledge about the type of event or affected region to help in effectively making relief decisions, e.g., which supplies will be needed on the ground. For instance, in order to effectively respond to Hurricane Laura's landfall during the COVID-19 pandemic, relief organizations source expertise on diseases, the region's demographic, and so forth. Unfortunately, acquiring the required data from a variety of data sources and locating experts is time consuming. 


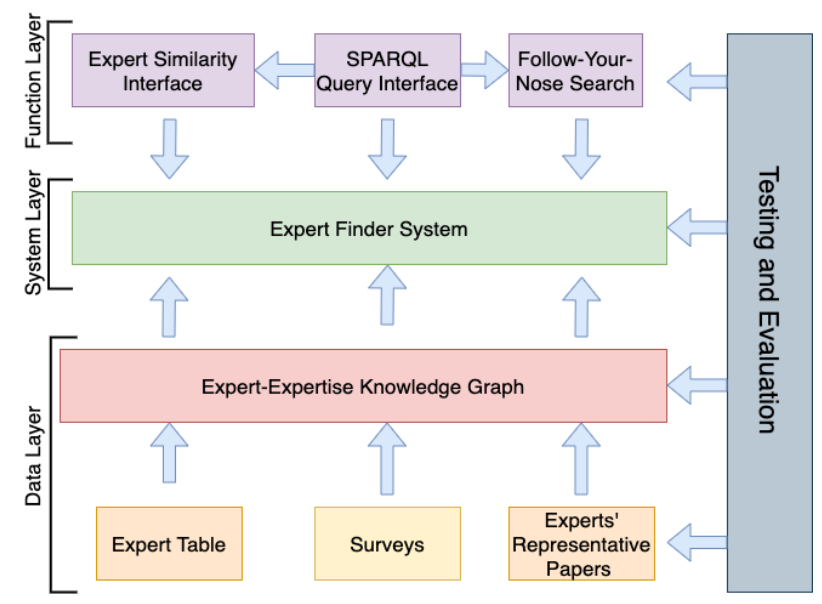

Figure 1: Overview of proposed Expert System Framework.

Existing expert finder systems (e.g., Expertise Finder ${ }^{1}$ ), mainly offer information about experts and their expertise. As such, this type of system may not meet the demand of relief organizations, due to a lack of connections to other relevant information (e.g., demographic information about affected areas). Furthermore, expertise in those systems is defined either as a field of study or a research topic. Yet relief organizations frequently attempt to acquire experts outside of academia. In response to Hurricane Laura, for example, they may consult local governors who are non-academic (hence having no area of study or research topic) but have first-hand experience dealing with individual disasters.

In order to assist a rapid response to disasters, our demo presents a knowledge graph (KG)-supported search engine - Expert Finder System ${ }^{2}$. We constructed an expandable and scalable expert knowledge graph (EKG) as the backbone of our system and integrated diverse datasets, such as hierarchically grouped expertise related to humanitarian relief, expert information (e.g., name and affiliation), disaster event, as well as geographic locations/trajectories of events and places. On top of EKG, we implemented three modes of information retrieval, i.e., follow-your-nose search, expert similarity search, and SPARQL query interface.

More concretely, Figure 1 shows an overview framework of our Expert Finder System. Data and function layer support the system layer with integrated data and humanitarian relief customized searching functions. Meanwhile, our system is being tested and evaluated by relief experts from Direct Relief ${ }^{3}$ using real world competency questions. Following sections will discuss about each with detailed examples.

\section{EXPERT KNOWLEDGE GRAPH (EKG)}

In this section, we briefly describe the procedure we used to construct our Expert Knowledge Graph (EKG), which is one of the key deliverable of our KnowWhereGraph [2, 4] project. We introduce the data sources, preprocessing procedures, and the schema that was designed for EKG.

\footnotetext{
${ }^{1}$ https://expertisefinder.com/

${ }^{2} \mathrm{http}: / /$ stko-roy.geog.ucsb.edu

${ }^{3}$ https://www.directrelief.org
}

\subsection{Data Acquisition and Preprocessing}

For the demo purpose, we use two publicly-accessible datasets from NOAA Storm Events Database ${ }^{4}$ and COVID-19 Mobility Data Network ${ }^{5}$. In addition we use responses collected from a customized online survey that was tailored to collecting expert information in specific interest areas (the developed survey can be accessed via Expert Survey).

The Storm Events Database contains event records (of over 50 event types, e.g., Flood, Drought and Hurricane) that temporally range from January 1950 to May 2021 in a structured csv format. This dataset includes information of where an event happened, when it started and ended, and its impacts (e.g., Crop Damage, Direct Death, and Indirect Injuries). The COVID-19 Mobility Data Network provides a seminal list of experts. We extended this list with additional attributes including expert name, affiliation, department, research interests, and publications that were mined from Google Scholar and respective personal web pages. We then grouped their research interests to generate two-tier expertise topics resulting in 19 different topic groups (e.g, Disaster Response Topic Group and Hazard Topic Group), each of which consists of several subtopics. For example, Hazard Topic Group comprises Drought Topic, Hurricanes Topic, Tropical Cyclones Topic, Typhoons Topic, and Wildfire Topic. The overview of all the topic groups and subtopics can be found in our demo ${ }^{6}$.

The linkage between an expert and an event is built based on the alignment between expertise topics and event types. For example, Hazard event is classified under Hazard topic. This alignment assists in identifying experts linked to specific expertise topics that match a disaster/hazard event.

\subsection{EKG Schemata}

Figure 2 illustrates the schema for our Expert Knowledge Graph, which consists of three major components: Expert, Expertise and Hazard Event. Mostly we reuse common ontologies, such as OWLTime[1] and SOSA[3]. Figure 3 and 4 shows the materialization of an expert and a hazard, respectively. As shown in Figure 3, each expert may be linked to expertise topics of various types, which are instances of kwg-ont:ExpertiseTopic. In reality, an expert can contribute to any event of a particular type that falls under their expertise topics. For example, if an expert has expertise in Drought Topic, then they are helpful in any drought-related events. A clear link between an expertise topic instance and an event type Class is drawn. Ontologically, we use the "punning" technique to build the instance-class connection in our schema, shown using kwgont:fallsUnderTopic in Figure 2. All the data was triplified using a Python library - rdflib $^{7}$ and the generated triples were stored in GraphDB.

\section{EXPERT SYSTEM OVERVIEW}

Three functions were combined to support the Expert Finder functionality. Specifically, follow-your-nose search facilitates relief specialists to freely explore instances about experts, places, hazards,

\footnotetext{
${ }^{4}$ https://www.ncdc.noaa.gov/stormevents/

${ }^{5}$ https://www.covid19mobility.org/

${ }^{6}$ http://stko-roy.geog.ucsb.edu/browse/\#kwg-ont:ExpertiseTopic

${ }^{7}$ https://github.com/RDFLib/rdflib
} 


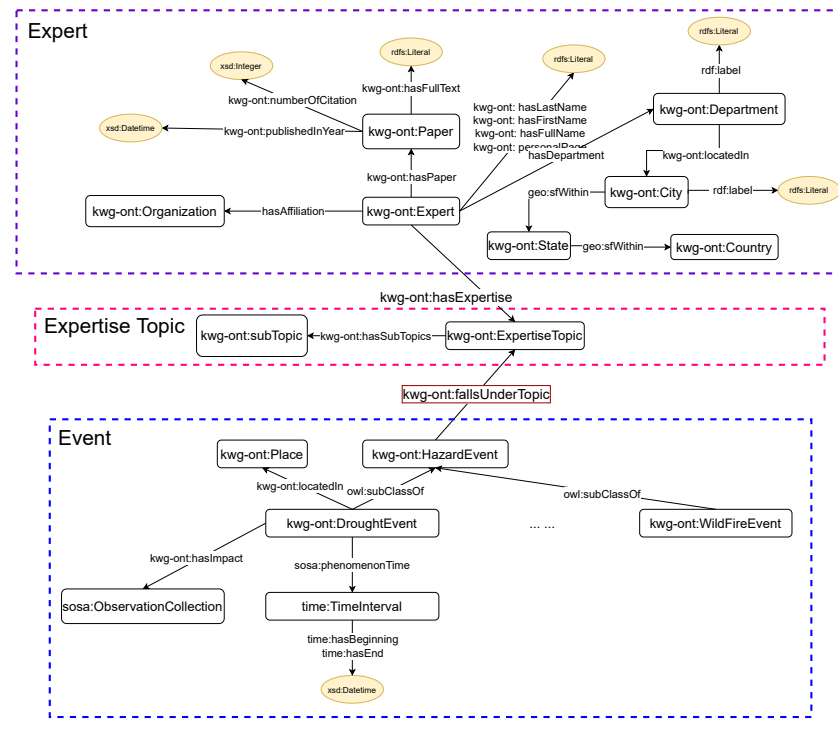

Figure 2: Schema of EKG.

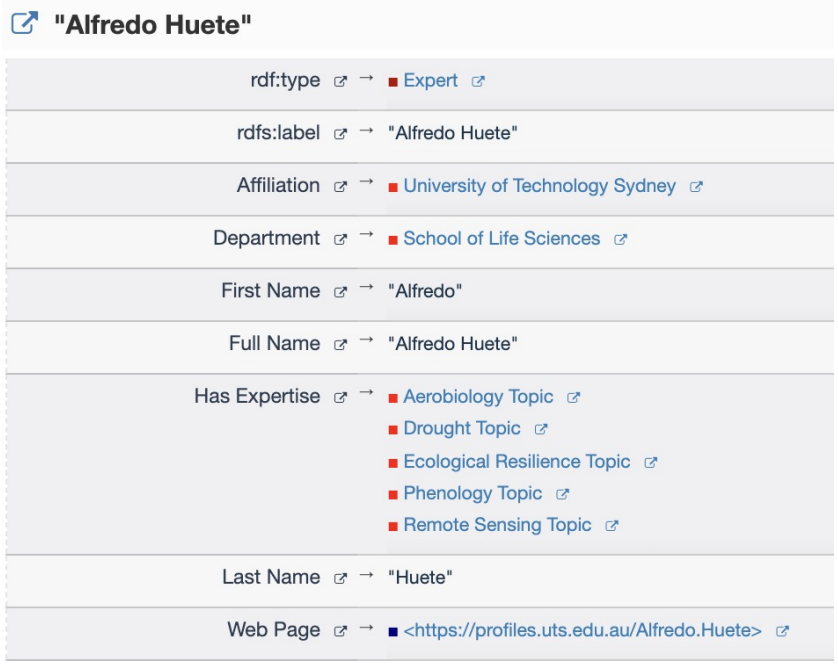

Figure 3: Instance data of an expert.

and their related information. The expert similarity interface enables quantitative similarity computation among experts based on their publications. A SPARQL query interface provides an API for these two interfaces to interact, while users with advanced knowledge in SPARQL can write queries to directly access the graph as well. The following examples show how each component works.

\subsection{Follow-your-Nose Search}

We designed a follow-your-nose style expert navigation tool, based on the PhuzzyLink framework [7] to assist relief specialists who are not familiar with Semantic Web techniques (e.g., SPARQL querying) efficiently explore the expert knowledge graph. This tool accommodates exploring the graph from three starting points. First, a relief

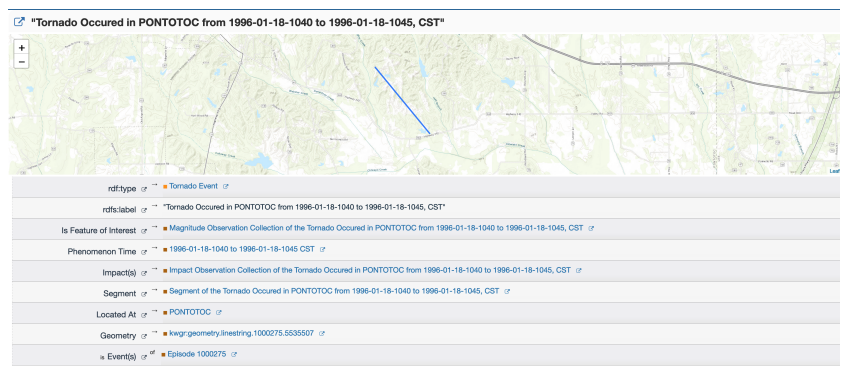

Figure 4: Instance data of a hazard event.

specialist can start by checking the expertise fields, such as computer science, biology, disease, and disaster responses, under which finer-grained subfields can be further explored, such as malaria, measles, and tuberculosis for disease. By clicking a subfield of interest, one can further find a list of experts who have expertise on it. Furthermore, by dereferencing the expert's URL, more detailed information of the expert, such as the affiliation, an expertise list, as well as the personal web page can be retrieved. The flow of such a search is illustrated in Figure 5. In a situation where specialists aim to find organizations related to a city or region, they can explore the graph from a list of places, and then view the set of organizations that are located within each place. From the organizations, affiliated experts, together with their detailed information can be further extracted. Finally, one can also start from a specific event type or instance of hazard (e.g., Drought Event) and eventually navigate to specific experts who have expertise in the topic (e.g., Drought).

\subsection{Expert Similarity Interface}

The above follow-your-nose search interface enables users to freely explore the Expert Knowledge Graph to locate experts. However, in some situations, relief specialists would want to quantitatively measure the similarity of experts. For example, when a known expert is not available, relief experts may want to find an alternative person who is most similar in terms of their area of expertise. To quantitatively measure such a similarity among experts, we adopt unsupervised document representation learning techniques [5]. More specifically, we first select $K$ representative papers (i.e., top 3 most cited and top 3 most recent papers) of each expert and merge these $K$ papers into a single document by expert. In the end, each expert is characterized by a merged document. Then we use Doc2vec ${ }^{8}$ to encode each document into a low-dimensional document embedding and measure the similarity between any two documents by using cosine similarity. Based on the similarity scores, we build an expert similarity search interface.

Figure 6 shows an example of searching result. When a user types "Bryan Grenfell", this interface will guess possible experts whose names are similar to the user's input. When the user picks one, this interface will show a ranked list of experts who share similar expertise with the input expert. Moreover, all the result are dereferencable. Namely, by clicking on any expert in the list, the user will be directed to the follow-your-nose interface which

\footnotetext{
${ }^{8}$ https://radimrehurek.com/gensim/auto_examples/tutorials/run_doc2vec_lee.html
} 


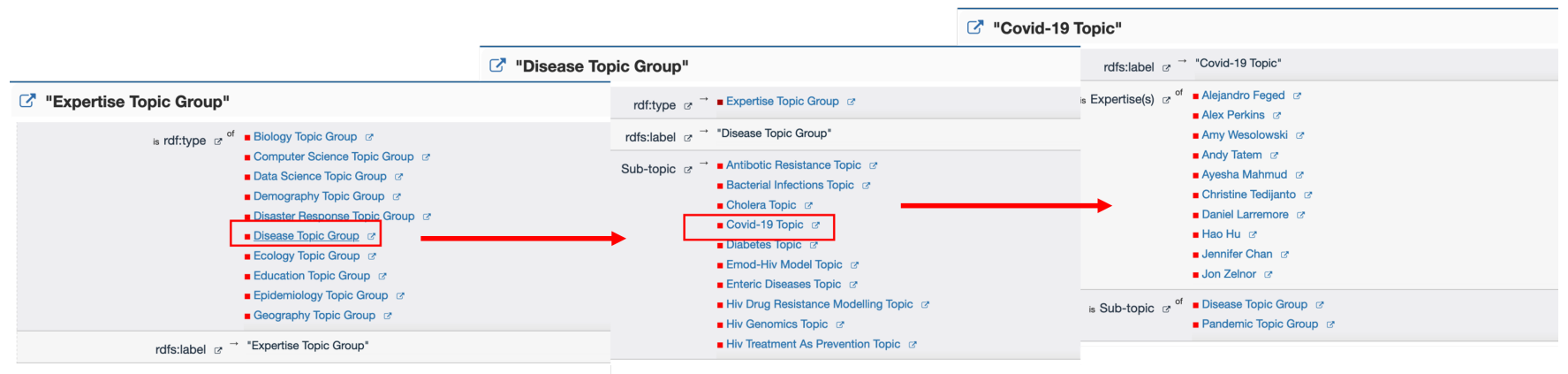

Figure 5: Example of follow-your-nose expert search from expertise topics.

then displays detailed information about this expert. In the future, we plan to refine the similarity metric by combining both natural language-based similarity and knowledge graph structure-based similarity [6].

\section{Expert Similarity Interface}

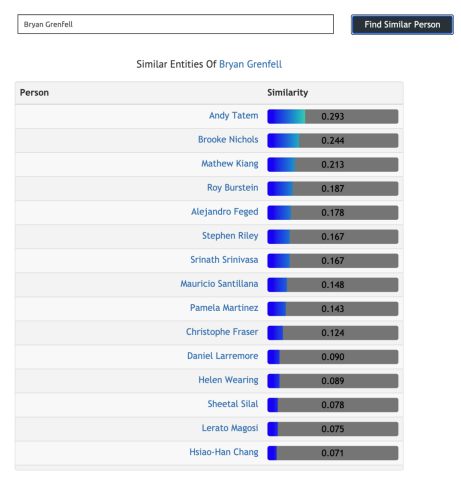

Figure 6: Example usage of expert similarity interface.

\subsection{SPARQL Query Interface}

In addition to the above introduced tools, we also provide a SPARQL endpoint for users to directly retrieve data. We mainly use this endpoint to evaluate the design of the proposed schemata by answering competency questions given by relief specialists from Direct Relief. One example of such a question is: "Who has expertise related to infectious diseases in the Sahel region?". Together with numerous other competency questions, the query and corresponding results can be found in our online repository ${ }^{9}$.

\section{CONCLUSION}

Rapidly identifying a matching expert based on their expertise in response to disasters is a non-trivial task, but key to disaster response. It requires the collection of data from multiple resources, clear schemata to integrate them, as well as a user-friendly interface to enable an easy and intuitive access to the data. Our Expert Finder

\footnotetext{
${ }^{9}$ https://github.com/KnowWhereGraph/Expert-System
}

System uses knowledge graphs and machine learning models to provide rich data as well as flexible search options for relief specialists to effectively make decisions. Aside from the used schemata, which are specifically designed to model expert and expertise for humanitarian relief, the described functionality, including the navigation and similarity-based search can be applied to other applications as well.

\section{ACKNOWLEDGMENTS}

This work was partially supported by the NSF award 2033521, "KnowWhereGraph: Enriching and Linking Cross-Domain Knowledge Graphs using Spatially-Explicit AI Technologies".

\section{REFERENCES}

[1] Jerry R Hobbs and Feng Pan. 2006. Time ontology in OWL. W3C working draft 27 (2006), 133.

[2] Krzysztof Janowicz. 2021. KnowWhereGraph Drives Analytics and Cross-Domain Knowledge. ArcUser (2021), 16-19.

[3] Krzysztof Janowicz, Armin Haller, Simon JD Cox, Danh Le Phuoc, and Maxime Lefrançois. 2019. SOSA: A lightweight ontology for sensors, observations, samples, and actuators. Fournal of Web Semantics 56 (2019), 1-10.

[4] Krzysztof Janowicz, Pascal Hitzler, Wenwen Li, Dean Rehberger, Mark Schildhauer, Rui Zhu, Cogan Shimizu, Colby Fisher, Ling Cai, Gengchen Mai, Joseph Zalewski, Lu Zhou, Shirly Stephens, Seila Gonzalez, Anna Lopez Carr, Andrew Schroeder, Dave Smith, Dawn Wright, Sizhe Wang, Yuanyuan Tian, and Zilong Liu. 2021. now, Know Where, KnowWhereGraph: A Densely Connected, Cross-Domain Knowledge Graph and Geo-Enrichment Service Stack for Applications in Environmental Intelligence. AI Magazine (2021). Under Review.

[5] Quoc Le and Tomas Mikolov. 2014. Distributed representations of sentences and documents. In International conference on machine learning. PMLR, 1188-1196.

[6] Gengchen Mai, Krzysztof Janowicz, and Bo Yan. 2018. Combining Text Embedding and Knowledge Graph Embedding Techniques for Academic Search Engines.. In Semdeep/NLIWoD@ISWC.77-88.

[7] Blake Regalia, Krzysztof Janowicz, and Gengchen Mai. 2017. Phuzzy. link: A SPARQL-powered Client-Sided Extensible Semantic Web Browser.. In VOILA@ ISWC. 34-44. 